\title{
Enhancements on multi-exposure LASCA to reveal information of speed distribution
}

\author{
D. Zölei-Szénási \\ zolei-szenasi.daniel@med.u-szeged.hu
}

S. Czimmer

T. Smausz

F. Domoki

B. Hopp

L. Kemény

F. Bari

I. Iványi
Department of Medical Physics and Informatics, University of Szeged, Korányi fasor 9., H-6720 Szeged, Hungary

Department of Optics and Quantum Electronics, University of Szeged, Dóm tér 9., H-6720 Szeged, Hungary

Department of Medical Physics and Informatics, University of Szeged, Korányi fasor 9., H-6720 Szeged, Hungary

Department of Optics and Quantum Electronics, University of Szeged, Dóm tér 9., H-6720 Szeged, Hungary

MTA-SZTE Research Group on Photoacoustic Spectroscopy, University of Szeged, Dóm tér 9., H-6720 Szeged, Hungary

Department of Physiology, University of Szeged, Dóm tér 10., H-6720 Szeged, Hungary

Department of Optics and Quantum Electronics, University of Szeged, Dóm tér 9., H-6720 Szeged, Hungary

Department of Dermatology and Allergology Albert Szent-Györgyi Medical Center, University of Szeged, Korányi fasor 6., H-6720 Szeged, Hungary

Department of Medical Physics and Informatics, University of Szeged, Korányi fasor 9., H-6720 Szeged, Hungary

Department of Optics and Quantum Electronics, University of Szeged, Dóm tér 9., H-6720 Szeged, Hungary

Laser Speckle Contrast Analysis (LASCA) has been proven to be a highly useful tool for the full-field determination of the blood perfusion of a variety of tissues. Some of the major advantages of this technique are its relatively high spatial and temporal resolution as well as its good or excellent accordance to Doppler systems. However, traditionally it is only able to report a single characteristic speed regarding to the actual range of interest. This might be misleading if multiple characteristic speeds are present (e. g. tremor and perfusion in skin) or if several kinds of tissues are mixed (e. g. parenchyma and vessels in brain). Here we present two relatively simple extensions of LASCA for these problems. The application of multiple autocorrelation functions (combined with the usage of multiple exposure times) can help in the separation of multiple characteristic speeds. We also present a useful method for the separation of information those originate from a mixture of different tissues. The latter method can be also implemented to single-exposure systems.

[DOI: http://dx.doi.org/10.2971/jeos.2015.15033]

Keywords: LASCA, speckle imaging, multi-exposure, multiple exposure times, speed distribution, multiple speed values

\section{INTRODUCTION}

Laser Speckle Contrast Analysis (LASCA) has been proven to be a highly useful tool for the full-field determination of the blood perfusion of a variety of tissues [1]-[6]. Some of the major advantages of this technique are its relatively high spatial and temporal resolution as well as its good or excellent accordance to Doppler systems. However, traditionally it is only able to report a single characteristic speed regarding to the actual range of interest. This might be misleading if multiple characteristic speeds are present (e. g. tremor and perfusion in skin) or if several kinds of tissues are mixed (e. g. parenchyma and vessels in brain). Here we present two relatively simple extensions of LASCA for these problems. The application of multiple autocorrelation functions (combined with the usage of multiple exposure times) can help in the separation of mul- tiple characteristic speeds. We also present a useful method for the separation of information those originate from a mixture of different tissues. The latter method can be also implemented to single-exposure systems.

$$
K=\frac{\sigma}{\mu}
$$

where $\sigma$ is the standard deviation and $\mu$ is the mean of the intensity values in a given $n \times n$ pixel window. For practical reasons, $n$ is generally chosen to be 5 or 7 [7]. The slower the motion is or the shorter the exposure time of the camera is, the sharper the speckle image becomes, hence, the higher the contrast is. Similarly, the faster the motion is or the longer the exposure time is, the more blurred the speckle image becomes and the lower the contrast is. The theoretical maximum and 
minimum of local contrast are 1 and 0 , respectively. Assuming an ergodic behaviour, the connection between the local contrast and the speed of motion is nonlinear:

$$
K^{2}(T)=\int_{0}^{T}\left(1-\frac{t}{T}\right) \cdot C^{2}(t) d t
$$

where $T$ is the exposure time, and $C(t)$ is the autocovariance function, which describes the temporal changes of the speckle pattern $[8,9]$. The contrast of natural speckle patterns cannot reach 1 , and in many cases the maximum value of contrast is significantly lower than $1[10,11]$, which results in the underestimation of speed values, if Eq. (2) is used. To handle this problem, the $\beta$ correction factor was introduced:

$$
K^{2}(T)=\int_{0}^{T} \beta\left(1-\frac{t}{T}\right) \cdot C^{2}(t) d t
$$

where $\sqrt{\beta}$ is the maximal value of contrast, which can be achieved by the given system [12]. If the sample contains still parts (e. g. skull or dura mater), the lower boundary of the contrast is also greater than $0[13,14]$ :

$$
K^{2}(T)=\int_{0}^{T} P_{1}^{2}\left(1-\frac{t}{T}\right) \cdot C^{2}(t)+P_{2}^{2} d t
$$

where $P_{1}^{2}+P_{2}^{2}=\lim _{T \rightarrow 0} K^{2}(T)$ and $P_{2}^{2}=\lim _{T \rightarrow \infty} K^{2}(T)$ characterize the scattering properties of the sample. It is remarkable that the use of Eq. (3) requires the application of a wide range of exposure times. One of the simplest autocorrelation functions is the Lorentzian one:

$$
C(t, \tau)=e^{\frac{t}{\tau}}
$$

where $\tau$ is the autocorrelation time of the speckle pattern, and is inversely proportional to the characteristic speed of motion of the examined sample $[15,16]$. The implementation of the Speckle Contrast Perfusion Unit (SCPU $=1 / \tau$ [17]) can simplify further work during the processing of Eq. (3) and Eq. (4):

$$
K^{2}(T)=\beta \frac{\tau^{2}}{2 T^{2}}[\exp (-2 T \cdot S C P U)-1+2 T \cdot S C P U],
$$

and

$$
\begin{aligned}
& K^{2}(T) \\
= & P_{1}^{2} \frac{\tau^{2}}{2 T^{2}}[\exp (-2 T \cdot S C P U)-1+2 T \cdot S C P U]+P_{2}^{2},
\end{aligned}
$$

respectively. Eq. (6) and Eq. (7) are able to accurately describe the speed of motion if only one characteristic speed is present, otherwise, they can only give an estimation of the average speed of motion. Unfortunately, the presence of one single characteristic speed is rare. If the set of exposure times is wide enough, the same speed distribution can be shown by a speckle system as by a Doppler system [18], however, this is hard to be related to multiple characteristic speeds. Nemati et al. published a different approach for the decoupling of artefacts in the speckle signal [19]. If the characteristic speeds (e. g. tremor and perfusion of skin) significantly differ from each other, the presumption of only a single one can lead to false results. Sections 2.1, 4.1, 5.1, and 6.1 address the determination of two or more characteristic speeds at the same time. In the case of cerebral or ocular tissue, large lateral fluctuations of SCPU is often present. As a consequence, an AOI (area of interest), whose size is large enough to suppress the statistical noise can contain contrast information of several characteristic speeds. Another severe problem is the small movement of the tissue during these measurements. Though these movements can be monitored and the AOI's can be shifted according to them, the sophisticated algorithms, which are needed for these measurements might be hard to implement. Sections $2.2,4.2,5.2$, and 6.2 address a simple and easy-to-implement method, which is able to describe the lateral distribution of $S C P U$, and, which can neglect the need for following the small and slow movements of the sample. Section ?? shows an alternative application of this method for the examination of rosacea.

\section{ENHANCED TOOLS FOR LASCA}

\subsection{Separation of different characteristic speeds by the use of multiple autocorrelation functions}

A typical example for the presence of two discrete and separable motions is the skin, where the blurring of perfusion of red blood cells and tremor are superposed. Classical LASCA methods can only reveal the change of the average speed of motion. Since normally SCPU of perfusion is greater by a magnitude than that of tremor, and their weights (the $P_{p}$ and $P_{t}$ values in Section 5.1) are comparable in the contrast data, the measured SCPU can much lower than the actual SCPU of perfusion. If ischemia is applied to the examined area, perfusion decreases and tremor might also change, moreover, their weights in the contrast also vary. Since most systems report the average SCPU as the SCPU of perfusion, these circumstances can lead to false results. This problem can be resolved if the system is able to handle two distinct autocorrelation functions. Eq. (7) can be extended to handle two distinct characteristic speeds:

$$
\begin{aligned}
K^{2}(T)= & \frac{P_{p}^{2}}{2 T^{2}\left(S C P U_{p}+S C P U_{t}\right)^{2}} \\
& \times\left\{\exp \left[-2 T\left(S C P U_{p}+S C P U_{t}\right)\right]\right. \\
& \left.-1+2 T\left(S C P U_{p}+S C P U_{t}\right)\right\} \\
& +\frac{P_{t}^{2}}{2 T^{2} S C P U_{t}^{2}}\left\{\exp \left[-2 T \cdot S C P U_{t}\right]\right. \\
& \left.-1+2 T \cdot S C P U_{t}\right\}+P_{s}^{2},
\end{aligned}
$$

where $p$ and $t$ indexes denote perfusion and tremor, respectively, while s stands for the skin surface.

\subsection{A simple approach for revealing information about the lateral distribution of speed in the sample}

Another interesting area of application is the examination of brain surface, where parenchymal tissue and vessels are mixed. A bottleneck of this sample is that slow motion of the cortex might occur, and as a result, the inspected areas can actually move out of the areas of interest (AOI's). Though there are intelligent algorithms, which can follow the movements 


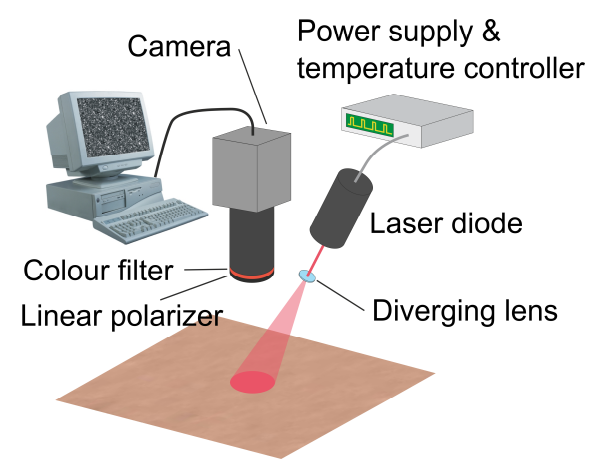

FIG. 1 Schematic drawing of the experimental setup.

of the sample, they are not easy to understand and implement. The method, which was developed by our group, can be easily implemented into most LASCA algorithms, and is able to visualize the lateral SCPU histogram of the inspected area. The SCPU histogram is based on the local SCPU values, which can be directly determined by the use of the local contrast values. Unfortunately, the local contrast values might be too noisy, especially if multiple exposure times are used (thanks to the propagation of uncertainty, [20]). To overcome the problem, we applied both temporal (windows size was 5 images having the same exposure time) and lateral (window size was $5 \times 5$ contrast pixels) running average to the contrast values. Afterwards, we calculated the SCPU values of each averaged pixels (involving each exposure times) and we built the histograms of SCPU values over the areas of interest (AOI's). This method can be highly useful e. g. in examination of cerebral microcirculation, when a mixture of different tissues has to be handled, or in the case of cutaneous diseases. The study of Abdurashitov et al. addresses the same problem and shows a different point of view of the question [21].

\section{EXPERIMENTAL SETUP}

The schematic figure of the experimental setup is shown in Figure 1. The sample was illuminated by the light of a laser diode (single-mode, $808 \mathrm{~nm}, 200 \mathrm{~mW}$ peak power) and was imaged by a PixeLINK PL-B741F industrial camera (FireWire, monochromatic, $1280 \times 1024$ maximal resolution). The laser diode was placed in a temperature controlled mount (Thorlabs LDM21) and was driven by a current and a temperature controller (Thorlabs LDC 220C, Thorlabs TED 200C, respectively). A colour filter was applied to reduce the effect of ambient illumination. A linear polarizer was also utilized, which was set to block the light, which was directly reflected by the surface of the sample.

\section{METHODS AND SAMPLES}

\subsection{Separation of different characteristic speeds by the use of multiple autocorrelation functions}

Here we present a representative measurement, which was performed on the skin of forearm of a healthy volunteer.
Wide range $(1,2,5,10,20,50,100,200,500 \mathrm{~ms})$ of exposure times was used to natively separate the data of perfusion and tremor. The applicability of the method in the case of a narrower set of exposure times $(1,2,5,10,20,50,100 \mathrm{~ms})$ was demonstrated by the application of post occlusive reactive hyperaemia. In this case, first, baseline was recorded. Then, occlusion was created by the cuff of a sphygmomanometer with a pressure which was higher than systolic pressure by 30 $\mathrm{mmHg}$. After the reperfusion a third measurement was also performed. Single-exposure monitoring was applied between the measurements, and each measurement was started after the stabilization of the contrast. The laser diode was operated in continuous-wave mode, the intensity of its light was set by the use of a variable neutral density filter. A Periflux 4000 contact Doppler system was used as reference. A detailed description of the protocol of measurement can be found in [17]. The most important benefit of this sampling method was that it could provide a wide range of exposure times, however, it was unable to show any perfusion data during the measurements and temporal changes during the multi-exposure measurements could not be retrieved.

\subsection{Revealing information about the lateral distribution of speed}

The data of a representative measurement is shown in this manuscript. In the case of the cortex of piglet the laser diode was operated in continuous-wave mode, the intensity of its light was set by the use of a variable neutral density filter [22]. The exposure time of $2 \mathrm{~ms}$ was used. The piglet was anaesthetized and the measurements were performed through a cranial window. A detailed description of the preparation of the animal and the protocol of measurement can be found in [22]. During the measurement on skin, the laser diode was operated in switching mode. This kind of operation made us possible to monitor the perfusion in real-time, while it provided the accuracy of the multi-exposure LASCA methods [23]. As the system could correct the effects of background illumination, the measurements could be performed by normal illumination, which highly enhanced the comfortness of the volunteer and provided an easier operation of the system. As the face of the volunteer was affected by rozacea, we chose two areas as near to each other as possible, which were positioned on a healthy and an ill area, respectively. The set of exposure times during this measurement was 1, 2, 5, 10, 20, 50 , and $100 \mathrm{~ms}$. A reference measurement of the skin of arm of a healthy volunteer is also presented for comparison. The detailed description of the reference measurement can be found in [20].

\section{RESULTS}

\subsection{Separation of different characteristic speeds by the use of multiple autocorrelation functions}

Figure 2 shows the raw speckle image of the skin of the forearm during the validation of the method. Area 'a' was covered by a piece of paper, and was used as reference. As it was nontransparent, the blurring, which was measured on it, could be 


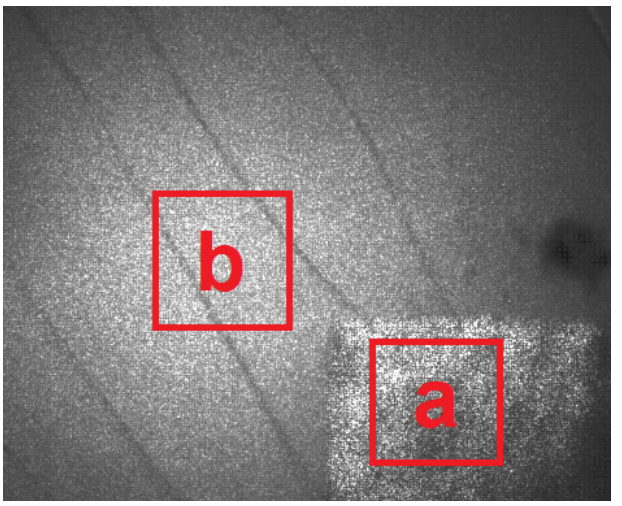

FIG. 2 Skin of the hand. a - reference area (paper; tremor only), b - skin (superposition of perfusion and tremor).

considered as the effect of tremor [24]. Area ' $b$ ' was selected on the skin, and contained the blurring information of tremor as well as the superposition of tremor and perfusion of red blood cells.

Figure 3 shows the function fittings based on single autocorrelation function and two autocorrelation functions. The $p$ and $t$, indexes stand for the perfusion, tremor, respectively, while $s$ denotes skin surface, respectively. Figure 3(a) shows the result of function fitting on the contrast values determined on the reference ('a') area. Figure 3(b) shows the result of function fitting on the contrast values determined on area ' $b$ ', utilizing single autocorrelation function and two autocorrelation functions.

As our results show, this method is able to separate the blurring caused by perfusion and tremor from each other. It is remarkable that the fitting, which uses two autocorrelation functions at the same time natively reports the very same 'perfusion rate' for the tremor, than the traditional fitting on the data which was registered on paper, if the range of exposure times was wide enough. The application of two autocorrelation functions also reveal that the true value of perfusion rate can be much higher than the average SCPU value which is calculated by the use of a single autocorrelation function.

\subsection{Lateral speed distribution of cortex}

This simple method can separate the flow or perfusion information which originate from different types of tissue. Figure 4(a) shows the contrast map of the cortex of a piglet and the AOI's, which cover the parenchyma (blue), a vessel (yellow), the area between them (green), and all of them (black). Figure 4(b) shows the speed histograms of these areas.

Calculation of speed distribution within the black area can help in the differentiation of the characteristic speeds of the three areas mentioned before, without the need for using tiny AOI's and sophisticated intelligent algorithms. As the speed of blood in the vessel is higher than that in the parenchyma, the data, which describes the flow rate of the vessel $(\sim 3.5-6.5$ $S C P U)$ and the perfusion rate of the parenchyma (1-3 SCPU), can be easily identified, respectively.
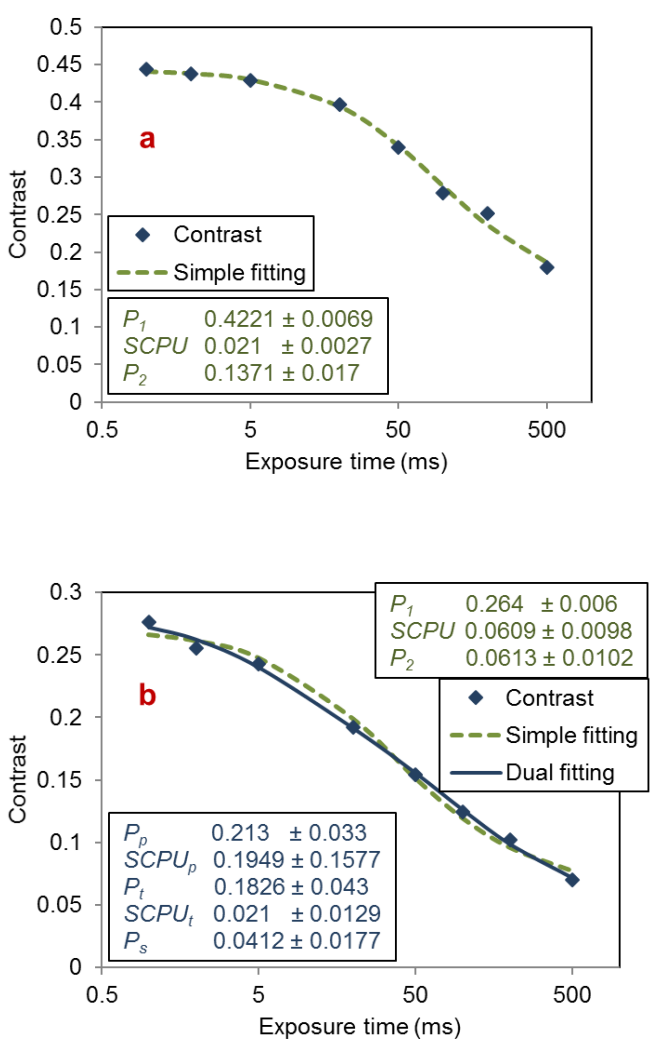

FIG. 3 Function fittings on the reference area (a; paper stuck on the skin, tremor only) and the skin ( $b$; perfusion and tremor). The use of two autocorrelation functions at the same time can help in the separation of tremor and perfusion. $a$ and $b$ markings also refer to the areas indicated in Figure 2 . The $p, t$, and $s$ indexes stand for the perfusion, tremor, and skin surface, respectively.

\section{DISCUSSION}

\subsection{Separation of different characteristic speeds by the use of multiple autocorrelation functions}

The use of multiple autocorrelation functions can be useful even if the set of exposure times is not wide enough for their direct implementation. In such a case, the 'perfusion index' of the paper has to be determined first. Since this is actually the value of tremor $\left(S C P U_{t}\right)$, it can be used in Eq. eqrefeq8 as a known fixed value. Table 1 summarizes our normalized results during baseline, occlusion and reperfusion of the arm of a healthy volunteer. The baseline values determined by the speckle and the Doppler systems were taken as $100 \%$, and the perfusion values during the occlusion and after the hyperaemia were normalized to the baseline. The error values are the normalised standard errors of the perfusion parameters during fitting.

The separation of perfusion and tremor can be critical during LASCA measurements, because presence at the same time can highly affect the readings during the occlusion of the arm or the leg. As Table 1 shows, the superposition of perfusion and tremor might not change as dynamically as the Doppler signal, which can provide misleading values of perfusion. However, their separation can highly enhance the correlation be- 


\begin{tabular}{|c|c|c|c|}
\hline$(\%)$ & Baseline & Occlusion & Reperfusion \\
\hline Relative $S C P U_{t}$ & $100 \pm 5.7730$ & $97.1378 \pm 6.2031$ & $106.2976 \pm 4.8858$ \\
\hline Relative $S C P U$ & $100 \pm 4.1468$ & $80.67514 \pm 5.0688$ & $104.6891 \pm 3.8562$ \\
\hline Relative $S C P U_{p}$ & $100 \pm 16.7394$ & $54.4260 \pm 18.0891$ & $95.6149 \pm 12.8958$ \\
\hline Relative $L D P U$ & 100 & 48.4636 & 102.9248 \\
\hline
\end{tabular}

TABLE 1 Relative changes of perfusion index of tremor $\left(S C P U_{t}\right)$ and the superposition of perfusion and tremor $(S C P U)$ determined by simple curve fitting. Relative changes of perfusion determined by dual autocorrelation function fitting with fixed $S C P U_{t}$. Relative changes of perfusion were also measured by a commercial contact Doppler system $(L D P U)$ as reference.
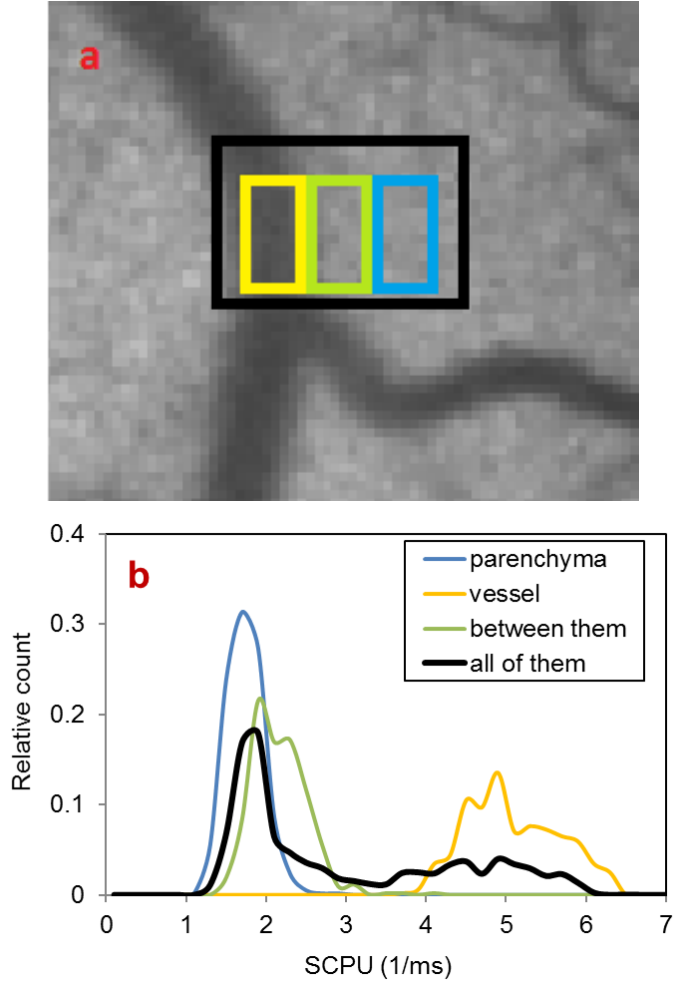

FIG. 4 Contrast map of the brain of a piglet. Blue frame - parenchyma, yellow frame vessel, black frame - area including both of them (a). Speed distribution of the areas mentioned above (b).

tween SCPU and LDPU values. If this method is applied to a multi-exposure LASCA system, it can perform these measurements in a very natural way, without the need of complex numerical corrections. If the range of exposure times cover more than 3 orders of magnitude, the system can perform the separation of tremor and perfusion in one step by simultaneously fitting the two contrast curves. Otherwise, a non-transparent sample (reference area) has to be taped on the skin, and its $S C P U_{t}$ value has to be determined. As the reference area and the skin are simultaneously monitored, the $S C P U_{t}$ can be used as a known parameter during the fitting of two autocorrelation functions on the data of skin.

\subsection{Lateral speed distribution of cortex}

The determination of speed distribution within a larger AOI can help in the differentiation of perfusion information of areas, which are mixed but have distinct perfusion rates. If asphyxia, ischemia, or any kind of vasoconstrictor or vasodilator is applied on the brain or the cortex, the vessels and the parenchyma might slightly move. If tiny AOI's are used, this

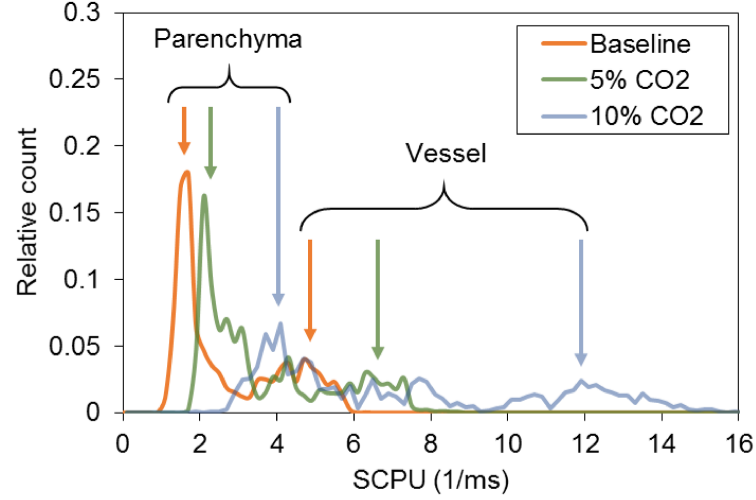

FIG. 5 Speed distribution of the cortex during baseline and the inhalation of air containing $5 \% \mathrm{CO}_{2}$ and $10 \% \mathrm{CO}_{2}$, respectively. The curves demonstrate that the determination of the speed distribution can help in the differentiation of the characteristic speeds over these areas, if all of them are included in the $\mathrm{AOI}$.

can result in false readings, since the vessel might move out the AIO, or a vessel can move in to an AIO which was originally positioned on the parenchyma. However, if a larger AOI is used for a vessel, which contains relatively large area of the parenchyma (like the black AOI on Figure 4), the slow displacement of the vessel does not affect the speed histogram in a large manner. Figure 5 shows the change of lateral speed distribution of the cortex during baseline and the inhalation of air containing $5 \% \mathrm{CO}_{2}$ and $10 \% \mathrm{CO}_{2}$, respectively. Though the position of the black AOI was locked during the measurement, its size was larger than the displacement of the vessel.

The increase of perfusion (approx. 120\% and 160\% in the parenchyma and the vein, respectively) can be generally evaluated as the response for the increased concentration of $\mathrm{CO}_{2}$ [22]. An interesting aspect of our results is that the size area, which is occupied by the parenchyma and the vessel, respectively, within the black AOI can also be also approximated by the calculation of the relative area under the specific part (approx. 1-3, 1.8-4.8, and 3-9 for the parenchyma, respectively, and approx. 3-6, 4.8-7.8 and 9-16 for the vessel, respectively) of the speed distribution curve. Performing this calculation results in $67 \%$ and $32 \%$ for the parenchyma and the vessel, respectively.

Another interesting area of application of the lateral speed distribution is the examination of skin. The methods which are currently applied on this field usually determine the average perfusion rate of the selected area (LASCA systems), or determine it only in a few volumes of $1 \mathrm{~mm}^{3}$ (Doppler sys- 


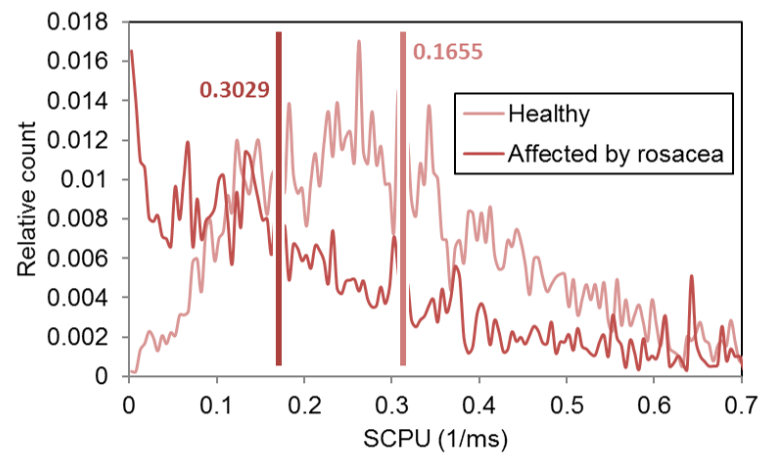

FIG. 6 Lateral speed distribution of a healthy area and one affected by rosacea. The speed distribution of the two areas indicates that rosacea leads to the decrease of the characteristic speed and to the dramatic change of the speed distribution of red blood cells. The figure also indicates the characteristic perfusion index values of the two areas.

tems). This information might be insufficient in several cases, since the physician can only see the average discrepancy of perfusion (with respect to a healthy area), or, in the latter case, the operator might not even have a representative image of the perfusion in the examined area. The visualization of SCPU histograms can help in the determination of the amplitude and the quality of the differences between two areas at the same time. Here we present an example for rosacea. The SCPU distribution was measured on a healthy area and on one which was affected by rosacea. We selected the two areas to make sure that they were as close as possible to each other to decrease systematic discrepancy of perfusion, which could have been caused by the different vascularity and sensitivity of the areas. Figure 6 shows that rosacea leads to a clear decrease of the perfusion rate and to the dramatic alteration of speed distribution of red blood cells. The figure also shows the average $S C P U$-values of the areas.

We performed a very similar measurement on the arm of a healthy volunteer before and during occlusion. The results can be seen on Figure 7.

The comparison of Figures 6 and 7 expressly indicates that in the former case the motion of red blood cells dramatically changed, however, they were not stopped. This can be a highly useful tool, since it can give a quantitative measure of the average change of perfusion (with respect to a healthy area) as well as qualitative information regarding to the way of change. Long-term variations of lateral speed distribution can also be used for the monitoring of the healing process of the patient, however, the evaluation of this aspect of the technique will require a full medical study.

\section{CONCLUSIONS}

Though LASCA is able to characterize the blood perfusion of many types of tissue, it is not able to provide little if any information about the speed distribution of the red blood cells. If the application of multiple exposure times is possible, LASCA

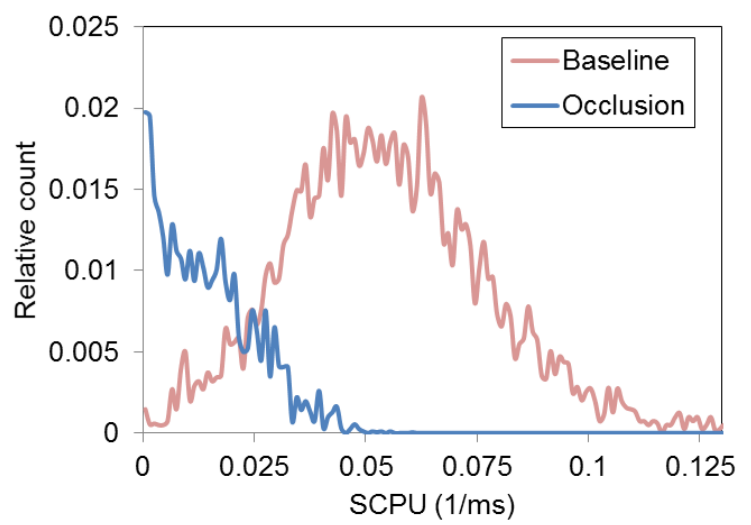

FIG. 7 Lateral speed distribution of the skin of forearm before and during occlusion. It can be clearly seen that during occlusion, red blood cells nearly stop moving.

can be extended to provide an enhanced description of the sample. The use of a wide range of exposure times, which has a scale of more than 3 magnitudes, can make the system able to use more than one autocorrelation functions at the same time. This technique can help in the separation of multiple kinds of motion of the sample (e. g. superposition of blood perfusion and tremor in the case of skin, or any other type of tissue affected by at least two types of motion). If only a narrower set of exposure times is available, the measurement of tremor by the help of a piece of non-transparent paper can be performed, and then, it can be used as a known fixed parameter during the use of dual autocorrelation functions for the accurate determination of perfusion. The differentiation of mixed parts of the examined sample (like brain surface, where parenchymal blood perfusion and vascular blood flow overlap each other) is achievable by proper sampling of the $K^{2}(T)$ and SCPU values across the images of the sample and the calculation of speed histograms of the inspected areas, since it can show the nominal speeds of the different types of tissue and the size of area, which they occupy. The examination of skin diseases can be also enhanced by the use of this method, as it can help in the determination of the amplitude and the quality of differences between healthy and ill areas. This technique can be used even if the range of exposure times is equal to or less than two magnitudes, or in the case of a single exposure time. One of the greatest properties of this technique is that it can be relatively easily implemented into nearly any multi-exposure or single-exposure systems.

\section{ACKNOWLEDGEMENT}

The project was partially funded by "TÁMOP-4.2.2.A$11 / 1 / \mathrm{KONV}-2012-0035$ - Investigation of the interactions of environmental and genetic factors in development of immune-mediated and cancer diseases" which was supported by the European Union and co-financed by the European Social Fund. This research was partially supported by the European Union and the State of Hungary, co-financed by the European Social Fund in the framework of TÁMOP 4.2.4. A/2-11-1-2012-0001 "National Excellence Program". Ferenc Domoki was supported by the János Bolyai Research Scholarship of the Hungarian Academy of Sciences. The 
publication was supported by TMOP-4.2.2.D-15/1/KONV2015-0024.

\section{References}

[1] A. K. Dunn, A. Devor, H. Bolay, M. L. Andermann, M. A. Moskowitz, A. M. Dale, and D. A. Boas, "Simultaneous imaging of total cerebral hemoglobin concentration, oxygenation, and blood flow during functional activation," Opt. Lett. 28(1), 28-30 (2003).

[2] A. Kharlamov, B. R. Brown, K. A. Easley, and S. C. Jones, "Heterogeneous response of cerebral blood flow to hypotension demonstrated by laser speckle imaging flowmetry in rats," Neurosci. Lett. 368(2), 151-156 (2004).

[3] Y. Aizu, K. Ogino, T. Sugita, T. Yamamoto, N. Takai, and T. Asakura, "Evaluation of blood flow at ocular fundus by using laser speckle," Appl. Optics 31(16), 3020-3029 (1992).

[4] M. Nagahara, Y. Tamaki, M. Araie, and H. Fujii, “Real-time blood velocity measurements in human retinal vein using the laser speckle phenomenon," Jpn. J. Ophthalmol. 43, 186-195 (1999).

[5] K. R. Forrester, I. C. Stewart, I. J. Tulip, C. Leonard, and R. C. Bray, "Comparison of laser speckle and laser Doppler perfusion imaging: measurement in human skin and rabbit articular tissue," Med. Biol. Eng. Comput. 40(6), 687-697 (2002).

[6] J. Stewart, R. Frank, K. R. Forrester, J. Tulip, R. Lindsay, and R. C. Bray, "A comparison of two laser-based methods for determination of burn scar perfusion: Laser Doppler versus laser speckle imaging," Burns 31(6), 744-752 (2005).

[7] D. D. Duncan, S. J. Kirkpatrick, and R. K. Wang, "Statistics of Local Speckle Contrast," J. Opt. Soc. Am. A 25, 9-15 (2008).

[8] J. D. Briers, and S. Webster, "Laser speckle contrast analysis (LASCA), "a nonscanning, full-field technique for monitoring capillary blood flow," J. Biomed. Opt. 1(2), 174-179 (1996).

[9] R. Bandyopadhyay, A. S. Gittings, S. S. Suh, P. K. Dixon, and D. J. Durian, "Speckle-visibility spectroscopy: a tool to study timevarying dynamics," Rev. Sci. Instrum. 76, 093110 (2005).

[10] S. J. Kirkpatrick, D. D. Duncan, and E. M. Wells-Gray, “Detrimental effects of speckle-pixel size matching in laser speckle contrast imaging," Opt. Lett. 33(24), 2886-2888 (2008).

[11] 0. P. Thompson, M. Andrews, and E. Hirst, "Correction for spatial averaging in laser speckle contrast analysis," Biomed. Express 2(4), 1021-1029 (2011).

[12] P. A. Lemieux, and D. J. Durian, “Investigating non-Gaussian scattering processes by using nth-order intensity correlation functions," J. Opt. Soc. Am. A 16, 1651-1664 (1999).
[13] T. Smausz, D. Zölei, and B. Hopp, "Real Correlation Time Measurement in Laser Speckle Contrast Analysis Using Wide Exposure Time Range Images," Appl. Optics 48, 1425-1429 (2009)

[14] A. B. Parthasarathy, W. J. Tom, A. Gopal, X. Zhang and A. K. Dunn, "Robust Flow Measurement with Multi-exposure Speckle Imaging," Opt. Express 16, 1975-1989 (2008).

[15] J. W. Goodman, "Statistical Properties of Laser Speckle Patterns," in Laser Speckle and Related Phenomena, 9-75 (Springer, Berlin/Heidelberg, 1975).

[16] A. F. Fercher and J. D. Briers, "Flow visualization by means of single-exposure speckle photography," Opt. Commun. 37(5), 326-330 (1981).

[17] D. Zölei, T. Smausz, B. Hopp, and F. Bari, "Multiple Exposure Time Based Laser Speckle Contrast Analysis: Demonstration of Applicability in Skin Perfusion Measurements," PCt0 1, 28-32 (2012).

[18] 0. B. Thompson, and M. K. Andrews, "Tissue Perfusion Measurements, Multiple-exposure Laser Speckle Analysis Generates Laser Doppler-like Spectra," J. Biomed. Opt. 15, 027015 (2010).

[19] M. Nemati, C. N. Presura, H. P. Urbach, and N. Bhattacharya, “Dynamic light scattering from pulsatile flow in the presence of induced motion artifacts," Biomed. Express 5(7), 2145-2156 (2014).

[20] D. Zölei, T. Smausz, B. Hopp, and F. Bari, “Self-tuning laser speckle contrast analysis based on multiple exposure times with enhanced temporal resolution," J. Eur. Opt. Soc.-Rapid 8, 13053 (2013).

[21] A. S. Abdurashitov, V. V. Lychagov, 0. A. Sindeeva, 0 . V. Semyachkina-Glushkovskaya, and V. V. Tuchin, "Histogram analysis of laser speckle contrast image for cerebral blood flow monitoring," Front. Optoelectron. 15, 10493 (2015).

[22] F. Domoki, D. Zölei, 0. Oláh, V. Tóth-Szüki, B. Hopp, and T. Smausz, "Evaluation of Laser-speckle Contrast Image Analysis Techniques in the Cortical Microcirculation of Piglets," Microvasc. Res. 83, 311-317 (2012).

[23] T. Smausz, D. Zölei, and B. Hopp, “Laser power modulation with wavelength stabilization in multiple exposure laser speckle contrast analysis," Proc. SPIE 8413, 84131] (2012).

[24] G. Mahé, P. Rousseau, S. Durand, S. Bricq, G. Leftheriotis, and P. Abraham, "Laser speckle contrast imaging accurately measures blood flow over moving skin surfaces," Microvasc. Res. 81(2), 183-188 (2011). 